\title{
Modelling and electro-optical testing of suspended particle devices
}

\author{
Ricardo Vergaz*, José-Manuel Sánchez-Pena, David Barrios, Carmen Vázquez, Pedro Contreras-Lallana
}

Grupo de Displays y Aplicaciones Fotónicas, Dept. Tecnología Electrónica, Universidad Carlos III de Madrid, C/Butarque, 15, E28911, Leganés, Madrid, Spain

\section{A R T I C L E I N F O}

Article history:

Received 1 August 2007

Received in revised form

23 May 2008

Accepted 18 June 2008

Available online 5 August 2008

Keywords:

Electro-optical materials

Optical properties

Organic materials

Electro-optical devices

Scattering particles

Thin films

Optical properties

Solar energy

\begin{abstract}
A B S T R A C T
Some smart windows make use of suspended particle devices (SPDs) which are made of charged rodshape particles that change their orientation in an applied electric field, thereby allowing transmittance control. In this work, the electro-optical behaviour of a commercial SPD is analyzed. Impedance analysis shows characteristics similar to those of a Randles circuit, and a modified equivalent circuit is proposed and experimentally validated. Intermediate levels of transmittance are obtained using a customized field programmable gate array (FPGA)-based electrical circuit. Finally, measurements are taken to check the applicability of the SPD device and control system in smart glazing or photonic applications.
\end{abstract}

\section{Introduction}

There are many products where controlled variable light transmission is desired: smart windows, skylights and sunshades for the architectural, aircraft, marine, automotive and appliance industries; sunglasses, goggles and other eyewear; self-dimmable automotive sunroofs, sun visors and rear-view mirrors; and flat panel information displays. Light transmission control can be obtained with chromogenic materials. The science and technology of these chromogenic materials has attracted growing interest during the recent years [1-3].

There are two main categories of chromogenic technologies, depending on the activation process: non-electrical and electrical. Within the first category the most relevant are photochromics, thermochromics and thermotropics. Three types of materials are mainly used in the second category: liquid crystals (LCS), electrochromic (EC) materials, and electrophoretic or suspended particle (SP) substances. Each type of material manages light transmittance control in its own way.

LCs dispersed in organic polymer matrices (polymer-dispersed liquid crystals (PDLCs)) may be prepared as flexible, wide-area thin films where LCs form microdroplets that strongly scatter visible light. When an AC field is applied perpendicularly to the film, its LC molecules change directions and its optical characteristics vary [4]. Electrochromism involves electroactive materials that present a reversible change in optical properties when electrochemically oxidized or reduced $[5,6]$.

The development of suspended electrophoretic particle devices spans many years. The first device based on suspended particle device (SPD) technology (also called "light valve") was invented by Dr. Edwin Land in the 1930s. The active layer of the device has needle-shaped or spherical particles suspended in an organic fluid or gel, and is laminated or filled between two transparent electrodes. Particles are suspended randomly when no voltage is applied, blocking the passing of light (see Fig. 1(a)). If a signal of alternate voltage is applied, the particles move their internal charges to a minimum energy state, rotating them and aligning as a set, permitting light to pass through the film (Fig. 1(b)). Typical transmission ranges are 20-60\%, with switching speeds of hundreds of milliseconds. The voltage required for the device to operate depends on its thickness, usually in a range between 20 and $150 \mathrm{~V} \mathrm{rms}$. In contrast to ECs, an electric field is required to keep the film transparent.

In this work, a commercial SPD has been analyzed in detail, both in electrical and optical response, based on a previous approach [7]. An improved electrical model is proposed and validated in order to permit understanding of the electro-optical switching of these devices. An electronic driver to control the device has been implemented and tested. Several conclusions about its operation are also presented. 


\section{Devices and experimental set-up}

The SPD test, with an active area of $28 \mathrm{~cm} \times 22 \mathrm{~cm}$ and a thickness of $300 \mu \mathrm{m}$, used for this work is a Cri-Regulite device supplied by CRICURSA (Cristales Curvados SA, Barcelona, Spain). CRICURSA [8] is a Licensee of Research Frontiers [9] for the SPDs. The film was made as detailed in Ref. [10], by means of a crosslinked polymer matrix with droplets of polyhalide particles suspended in a liquid suspension distributed in it. The first step is the synthesis of a matrix resin with pendant phenyls. A photoinitiator was dissolved in $1 \mathrm{ml}$ of tetrahydrofuran (THF) and added to $1 \mathrm{~g}$ of the matrix resin. The combination was mixed and left in a vacuum oven at $90^{\circ} \mathrm{C}$ under reduced pressure for $1 \mathrm{~h}$. A suspending polymer and a paste of pyrazine dicarboxylic acid polyiodide crystals were added to $0.182 \mathrm{~g}$ of a suspending polymer with a molecular weight that leads to the decreasing viscosity of the capsules containing the crystals and to the reduction of their response time to an externally applied electrical voltage [10].

The emulsion was spread over an indium tin oxide-coated glass plate using a glass rod and exposed to UV radiation for $30 \mathrm{~s}$ in order to cure it. Curing was carried out with a second indium tinoxide-coated glass plate on top of the emulsion. Refractive indices

a

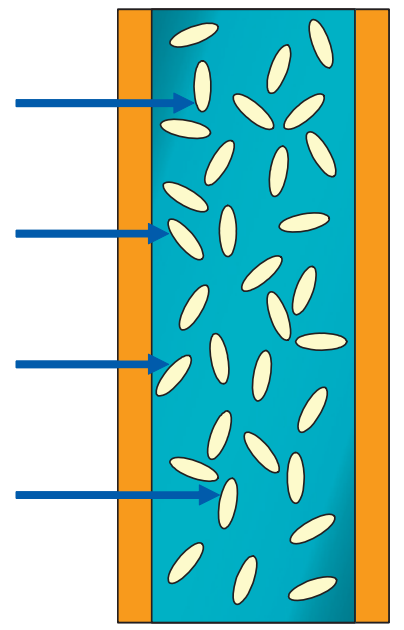

b

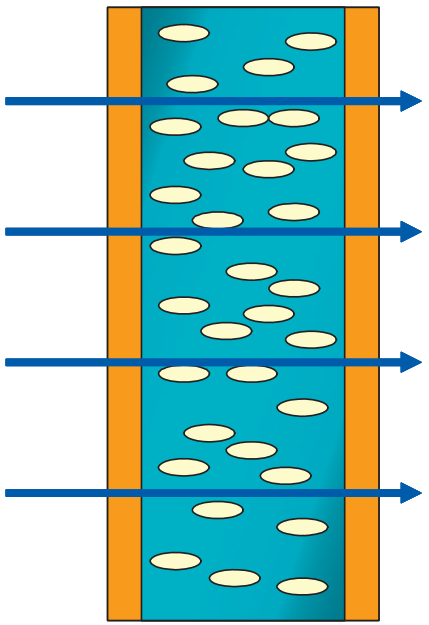

Fig. 1. Working principle of a suspended particle device: (a) operation with no applied voltage and (b) operation with applied voltage. of the droplets and the matrix components in the film matched to within 0.005 , and the droplets were specified around $1 \mu \mathrm{m}$ in size, both items leading to a low-scattering and blue-tone device, as can be seen in Fig. 2.

Fig. 3 shows a microscopic photograph of the main layer, in which the spherical particles are suspended. A mean diameter of $8.9 \mu \mathrm{m}$ is obtained in the micro-droplets originating from the manufacturing process. The homogeneity in the droplet distribution, measured through photographs such as the one shown, resulted in a density of 1050 droplets $/ \mathrm{mm}^{2}$. By means of microscope focusing, a layer depth of around $30 \mu \mathrm{m}$ is determined.

Impedance analysis was carried out using a Solartron 1260 analyzer, capable of scanning between $0.5 \mathrm{mHz}$ and $32 \mathrm{MHz}$, with a voltage bias between $\pm 40.95 \mathrm{~V}$. It can measure impedances of up to $1014 \Omega$. The optical response of the device was measured during switching, using a lamp with an emission similar to the A illuminant, and an Acton Research monochromator with a Hamamatsu photomultiplier tube, a system with $5 \mathrm{~nm}$ resolution and $1 \%$ error in radiance measurement. Transmittance measurements are taken through an optical fibre bundle in close proximity to the SPD surface, with a numerical aperture of 0.22 . The acceptance angle is $12.7^{\circ}$, which means that the main contribution to measured transmittance is by direct, not diffused (scattered) light.

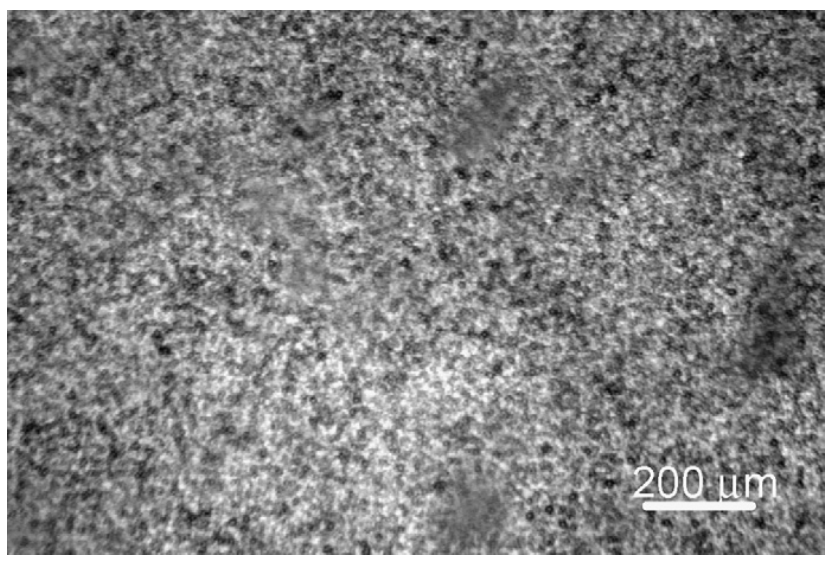

Fig. 3. Microscopic photograph of the SPD balls obtained focusing between the two contact layers. a

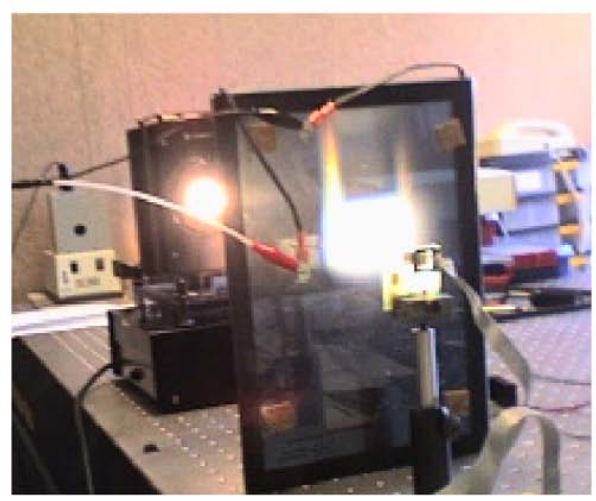

$b$

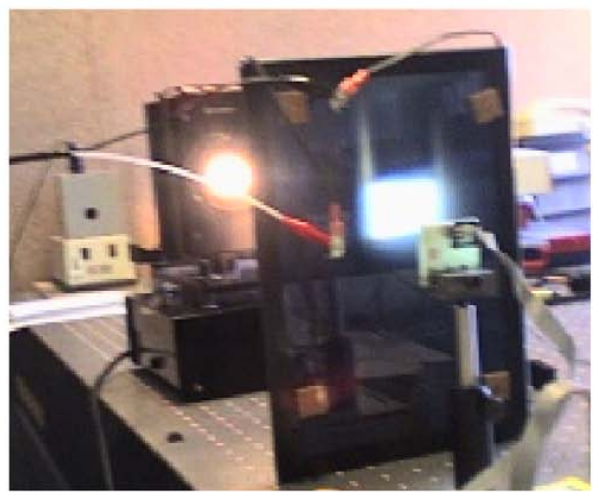

Fig. 2. Photograph of the SPD and the measurements setup: (a) with applied voltage and (b) without applied voltage. 
A field programmable gate array (FPGA)-based customized electronic driver was designed and implemented for addressing the SPD. This driver can be used, in principle, with devices of arbitrary size, except for the output power stage which largely depends on panel consumption. This has been measured to be about $5 \mathrm{~W} / \mathrm{m}^{2}$ in our current working conditions.

\section{Results and discussion}

\subsection{SPD electrical modelling}

Impedance spectroscopy is the main tool used to obtain information about the electrical response of this kind of device. According to the structure of the device in this work, the electrical equivalent circuit that can reproduce electrical behaviour of the SPD in real operation conditions is supposed to be based on a regular Randles circuit (resistance in series with a shunt capacitor-resistance set). This is the circuit most widely used for representing a two-layer capacitor-like device [11].

This is the equivalent circuit proposal made for our test particle suspended devices (Fig. 4(a), inset, up). Each electrical element accounts for some parameter involved in the fabrication of the SPD: the resistance (Rct) reflects the charge transfer insertion reaction, and is paired in parallel with a capacitor that stands for the double-layer capacitance $(\mathrm{Cdl})$ associated with the interfaces between charged zones. These elements are the main parts of the usual Randles circuit. The resistance (Rs) usually corresponds to the resistive effect of electrical contacts and it is in series with the rest of the set. But there is also a diffusion of charges in our devices, and hence a Warburg impedance $(W)$ should be added in series with Rct.

Rct was suppressed in the analysis because of its poor results in comparison with the rest of the components in the equivalent circuit: errors in Rct higher than $100 \%$ in the fit, and variations under $1 \%$ in the rest of the components if Rct is suppressed, lead to its elimination. The Warburg impedance thus absorbs the charge transfer resistance effect. $\mathrm{Cdl}$ is transformed into a simple capacitor.

The analysis of the parameters retrieved by fitting the impedance measurements to this circuit through an inversion least-squares method can serve as a way to make a manufacturing feedback improving process. In the same Fig. 4, a plot of the measured impedance can be seen, both in magnitude and phase angle. The decay in magnitude, mainly logarithmic for low frequencies, and the phase curling around $-90^{\circ}$ show a clear capacitive effect coming from the Cdl capacitance. Nevertheless, the clearly bi-lobular phase shape also shows the effect predicted by the Warburg element.

a

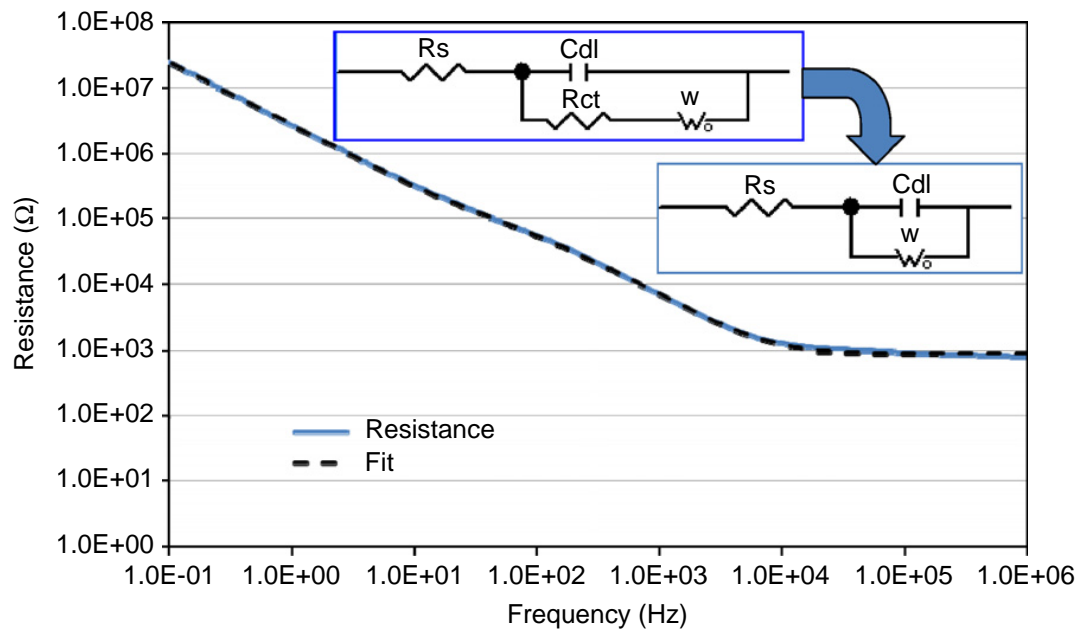

b

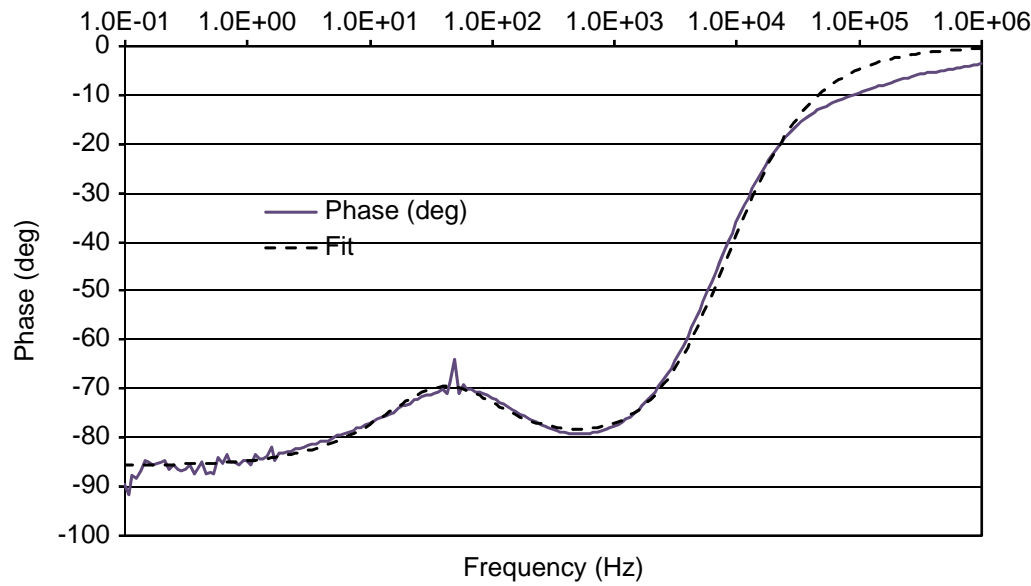

Fig. 4. Bode plots of the magnitude in resistance (a) and phase in degrees (b) of the impedance (solid lines) and best fitting (dashed lines) for the proposed equivalent circuit of the SPD (insets in (a): up, Randles circuit; down, the modified applied one). 
Table 1

Best fitting values and mean relative errors for the proposed equivalent circuit of the SPD

\begin{tabular}{llllll}
\hline Equivalent circuit & $\mathrm{Rs}$ & $\mathrm{Cdl}$ & $W-R$ & $W-T$ & $W-P$ \\
\hline Values & $919 \Omega$ & $20.5 \mathrm{nF}$ & $401.6 \Omega$ & $0.013 \mathrm{~s}$ & 0.465 \\
Errors (\%) & 0.5 & 0.7 & 3.3 & 4.2 & 0.4 \\
\hline
\end{tabular}

The fitted data for this device have the magnitudes (data and errors) shown in Table $1 . W-R, W-T$ and $W-P$ are integrating parameters of the Warburg impedance element, a resistance, time constant and power exponent respectively (Eq. (1)):

$\mathrm{Wo}=R \frac{\operatorname{ctnh}\left[(\mathrm{j} \omega T)^{P}\right]}{(\mathrm{j} \omega T)^{P}}$

where $R$ is $W-R, P$ is $W-P$ and $T$ is $W-T$. Warburg impedance stands for diffusion of charges; $W-R$ is the electrical resistance seen by these charges, $W-P$ is near 0.5 for a finite element, and $W-T$ is dependent on effective diffusion thickness and the effective diffusion coefficients of the particles. A higher $W-T$ stands for longer diffusion paths of charges. In this sense (Eq. (2)),

$W-T=\frac{L^{2}}{D}$

where $L$ is the effective diffusion thickness, and $D$ the effective diffusion coefficient of the particle. This element reflects the solution of the one-dimensional equation of particle diffusion.

As can be seen in Table 1, the errors in retrieved parameters are not higher than $4 \%$. This is a permissible error, because components used in cheap electronic driving circuits usually have tolerances of $\pm 5 \%$, and power consumptions that can be computed with these data can reach up to an $8 \%$ error. Thus, the model can be considered as consistent with the acquired experimental data.

Physico-chemical considerations lead us to interpret Rs as the resistance of the contacts-droplets set, Cdl as the capacitance formed by the contacts-emulsion set, and $W-R, W-T$ and $W-P$ the parameters involved in the charge movement inside the droplets. Indeed, Rct disappears because its effect is assumed at $W-R$. Cdl is, therefore, a simple capacitance because the device is as simple as that from a global electrical point of view. A Warburg impedance can stand for movement parameters of charges inside the droplets.

\subsection{Electro-optical testing}

Optical direct transmission in the bleached state $-<25 \%$-is still poor for many photonic applications. However, a systematic and extensive characterization of the test SPD was performed in order to provide some clues for improving specific parameters involved in the manufacturing process as well as to propose some potential new applications.

A measurement of the transmittance change at $600 \mathrm{~nm}$ with the applied voltage is shown in Fig. 5(a), in which a quadratic relationship can be established between both magnitudes, with a correlation coefficient higher than 0.99 .

No relevant changes are measured for signals with amplitudes over $100 \mathrm{~V} \mathrm{rms}$, where the SPD reaches the optical saturation level. This electro-optical characteristic is very useful in designing the right addressing waveforms for this type of devices. Based on this electro-optical behaviour, the electronic FPGA-based driver mentioned above has been implemented, which is able to stabilize 10 different electrically activated optical transmission levels for the SPD. As can be seen in Fig. 5(b), the best contrast in wavelength

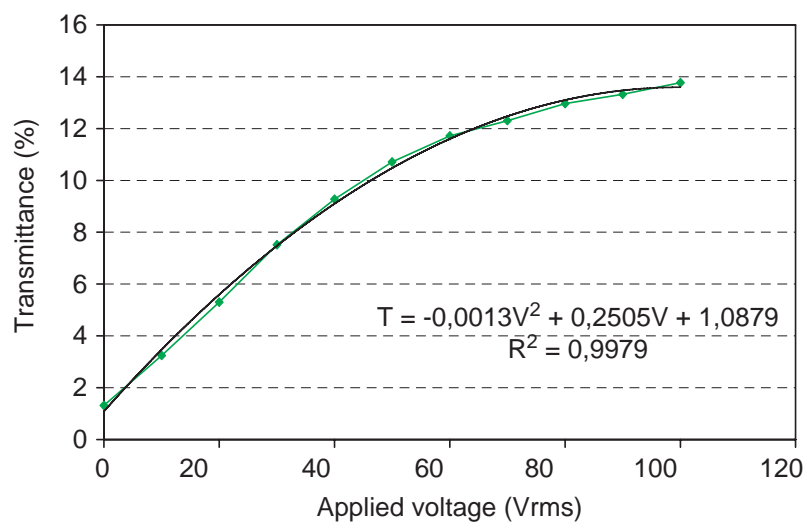

b

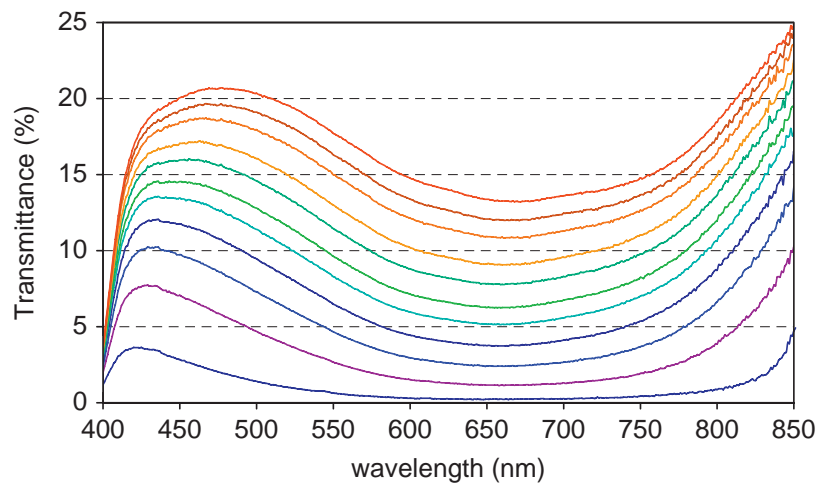

Fig. 5. Transmittance measured at $600 \mathrm{~nm}$ as a function of the applied rms voltage (dots). Line and inset: least squares fit to a quadratic relationship between both magnitudes. (b) Transmittance spectra of the device at the different tested FPGA voltage levels.

can be seen at around $480 \mathrm{~nm}$. The dark-colored state is a product of the low transmittance, but diffuse radiation is not measured. Being particles of $8.9 \mu \mathrm{m}$ diameter, Mie scattering is expected to be produced and diffuse transmittance can be important. The different ratio between the blue and the red intervals of the spectrum, defined at $440-490$ and $600-700 \mathrm{~nm}$, respectively, makes a bluer coloration, especially at low or not applied voltages (ratios of 9:1 in the integrated mean transmittance). Increasing the transmittance in the whole spectrum with higher voltages makes the device more transparent.

In order to check the SPD capability to be used as a smart window providing a sufficient number of intermediate transmission levels, an experimental procedure was performed using the monochromator. The transmittance evolution of the SPD was measured at $600 \mathrm{~nm}$ wavelength while applying square pulses with different amplitudes supplied from the implemented electronic driver. Up to 10 intermediate optical levels were obtained using this technique, as shown in Fig. 6(a). A quasilinear variation in the optical transmission maxima was obtained by applying appropriate amplitudes in the addressing pulses.

The next measurements are of the response times. To achieve them, the devices must be addressed with high voltage steps. A sinusoidal signal is needed to drive them, and the potential steps are obtained through steps in rms (root mean square) value, from 0 to 140 Vac. Measured rise and fall times (Fig. 6(b)), as they pass from $10-90$ and $90-10 \%$ of the transmittance change interval, slightly disagree with the data sheet supplied from the manufacturer (both ranging in hundreds of milliseconds for each time). 
a

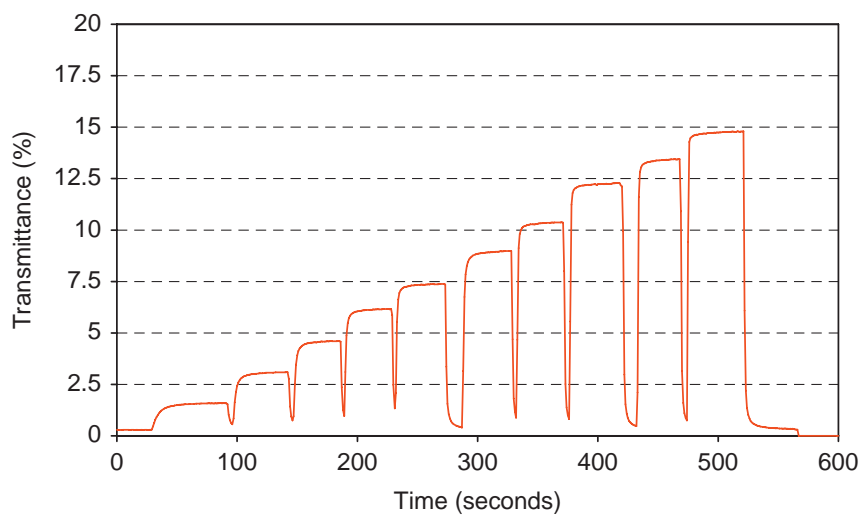

b

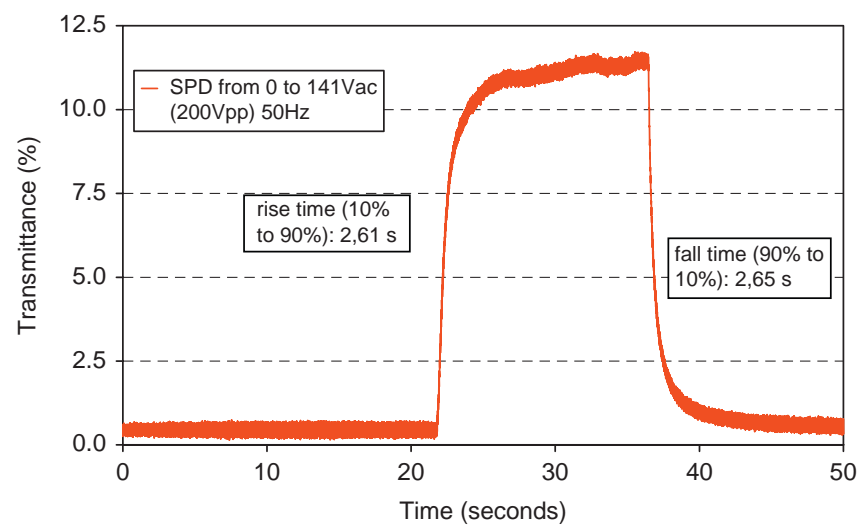

Fig. 6. SPD transmittance evolution at $600 \mathrm{~nm}$ wavelength while applying square pulses with different amplitudes. (a) All the levels shown. (b) Temporal details for level $\# 7$.

Finally, it is noteworthy that the device could be cycled $<1000$ switches before breakdown, a significantly poorer performance than expected by the company datasheet. Stresses caused by abrupt changes in the applied voltages should be studied further to assess conclusions about cyclability.

\section{Conclusions}

In this work, a SPD (manufactured by Cricursa) has been modeled and electro-optically tested. An equivalent circuit made for reproducing the measured impedance data has been proposed and is concluded to be consistent with them. This circuit shows the effects of the double-layer capacitance and charge transfer processes. These effects can be simulated through this equivalent circuit to improve the power consumption and also to predict time performance of the device. The reduction in the Rs-Cdl set values is necessary to obtain this power improvement. This can be achieved by reducing the thickness of the active layer (reducing (dl) or by obtaining an enhanced conductivity droplet type (reducing Rs). The physical parameters that can affect the electrooptical response of SPD can be monitored through this new equivalent circuit and related to critical parameters of the manufacturing process. Moreover, a complete set of systematic electrical and optical measurements have been carried out to evaluate potential applications of SPDs as smart windows among others.

Switching times are still poor for photonic applications such as optical filters, variable optical attenuators and switches, but are sufficient for smart glazing applications, which usually need only a few changes of state per day. Along these lines, a specific driver has been implemented, with a linear response and easily adaptable to several illuminating conditions. Further improvements in the substances, on the other hand, are needed to obtain better transmittance ranges. Thus, future research should focus on new materials and manufacturing processes.

\section{Acknowledgements}

This research was partially supported by funds from the Spanish Ministry of Science and Technology (Project PTR950940.01.OP), and by Comunidad de Madrid (Project FACTOTEMCM:S-0505/ESP/000417). The authors are grateful to CRICURSA for supplying the characterized device.

\section{References}

[1] C.M. Lampert, Smart switchable glazing for solar energy and daylight control, Sol. En. Mat. Sol. Cells 52 (1998) 207.

[2] W. Feng, S.H. Patel, M.-Y. Young, J.L. Zunino, M. Xanthos, Smart polymeric coatings-recent advances, Adv. Polymer Tech. 26 (2007) 1.

[3] S.K. Deb, Opportunities and challenges in science and technology of WO3 for electrochromic and related application, Sol. En. Mat. Sol. Cells 92 (2008) 245.

[4] Marialuigia Macchione, Giovanni De Filpo, Fiore P. Nicoletta, Giuseppe Chidichimo, Photochromic reverse mode polymer dispersed liquid crystals, Liq. Crys. 32 (3) (2005) 315.

[5] C.G. Granqvist, Electrochromic tungsten oxide films: review of progress 1993-1998, Sol. En. Mat. Sol. Cells 60 (3) (2000) 201.

[6] C. Pozo-Gonzalo, D. Mecerreyes, J.A. Pomposo, M. Salsamendi, R. Marcilla, H. Grande, R. Vergaz, D. Barrios, J.M. Sánchez-Pena, All-plastic electrochromic devices based on PEDOT as switchable optical attenuator in the near IR, Sol. En. Mat. Sol. Cells 92 (2) (2008) 101.

[7] R. Vergaz, J.M.S. Pena, D. Barrios, I. Pérez, J.C. Torres, Electrooptical behaviour and control of a suspended particle device, Opto-Electron. Rev. 15 (3) (2007) 154.

[8] 〈http://www.cricursa.com/ $\rangle$.

[9] 〈http://www.refr-spd.com/ $\rangle$.

[10] S. Chakrapani, S.M. Slovak, R.L. Saxe, B. Fanning, SPD films and light valves comprising same, Research Frontiers-US Patent 6416827, 2002. See < http:// www.patentstorm.us/patents/6416827-description.html $>$.

[11] Evgenij Barsoukov, J. Ross Macdonald (Eds.), Impedance Spectroscopy: Theory, Experiment, and Applications, Wiley-Interscience, Amsterdam, ISBN 0471647497, 2005. 